\title{
Operation of trans-thylakoid thiol-metabolizing pathways in photosynthesis
}

\author{
Mohamed Karamoko ${ }^{1,2}$, Stéphane T. Gabilly ${ }^{1,2+}$ and Patrice P. Hamel ${ }^{1,2 *}$ \\ 1 Department of Molecular Genetics, The Ohio State University, Columbus, OH, USA \\ ${ }^{2}$ Department of Molecular and Cellular Biochemistry, The Ohio State University, Columbus, OH, USA
}

\section{Edited by:}

Jose A. Traverso, Consejo Superior de Investigaciones Científicas, Spain

\section{Reviewed by:}

Jose A. Traverso, Consejo Superior de Investigaciones Científicas, Spain

Eevi Rintamäki, University of Turku,

Finland

\section{${ }^{*}$ Correspondence:}

Patrice P. Hamel, Department of

Molecular Genetics, The Ohio State University, 500 Aronoff Laboratory

318 West 12 th Avenue, 43210

Columbus, $\mathrm{OH}, \mathrm{USA}$

e-mail: hamel.16@osu.edu

${ }^{\dagger}$ Present address:

Stéphane T. Gabilly, Department of Plant and Microbial Biology, University of California, 441 Koshland Hall, Berkeley, CA 94720-3120, USA.
Thiol oxidation to disulfides and the reverse reaction, i.e., disulfide reduction to free thiols, are under the control of catalysts in vivo. Enzymatically assisted thiol-disulfide chemistry is required for the biogenesis of all energy-transducing membrane systems. However, until recently, this had only been demonstrated for the bacterial plasma membrane. Long considered to be vacant, the thylakoid lumen has now moved to the forefront of photosynthesis research with the realization that its proteome is far more complicated than initially anticipated. Several lumenal proteins are known to be disulfide bonded in Arabidopsis, highlighting the importance of sulfhydryl oxidation in the thylakoid lumen. While disulfide reduction in the plastid stroma is known to activate several enzymatic activities, it appears that it is the reverse reaction, i.e., thiol oxidation that is required for the activity of several lumen-resident proteins. This paradigm for redox regulation in the thylakoid lumen has opened a new frontier for research in the field of photosynthesis. Of particular significance in this context is the discovery of trans-thylakoid redox pathways controlling disulfide bond formation and reduction, which are required for photosynthesis.

Keywords: thylakoid lumen, photosynthesis, disulfide, thioredoxin
Thiol-disulfide chemistry refers to the oxidation of thiols (sulfhydryls) into disulfides and also the reversible reaction, i.e., the reduction of the disulfides to free thiols. In vivo, both chemical reactions are catalyzed by dedicated enzymes in cellular compartments where disulfide bonds and reduced thiols in protein targets need to be maintained at all times. Thiol-disulfide chemistry has been best studied in bacteria where it is required for the biogenesis of the periplasmic compartment (Denoncin and Collet, 2013). It has now become apparent that catalyzed thiol-disulfide reactions also operate in the mitochondrial intermembrane space (IMS) and the thylakoid lumen, which are topologically equivalent to the bacterial periplasm. In mitochondria, the Mia40/Erv1 proteins were discovered to be key enzymes of a disulfide relay system driving the import of cysteine-rich proteins into the IMS by an oxidative folding mechanism (Herrmann and Riemer, 2012). The thylakoid lumen has long been viewed as a vacant compartment, with the exception of the oxygen evolving complex (OEC) and some of the redox carriers (e.g., cytochrome $f$, Rieske, and plastocyanin-or its substitute cytochrome $c_{6}$ ) involved in electron transfer during the light reactions of photosynthesis. This dogma has now been revised with the realization that numerous molecules reside in the thylakoid lumen in addition to the previously known photosynthetic proteins. Proteomics studies revealed that the lumenal proteome $(\sim 80-200$ proteins $)$ is far more complicated than initially anticipated and includes proteases, chaperones, isomerases, redox enzymes, and other proteins of unknown activities (Kieselbach and Schröder, 2003). It is possible that the proteins revealed by proteomics regulate photosynthesis or other yet-to-be discovered processes unrelated to photosynthesis.
A recent study demonstrated that lumenal PPD5 is not involved in photosynthetic electron transfer reactions, but rather controls the synthesis of strigonolactone, a plant hormone regulating axillary bud formation (Roose et al., 2011). There is mounting evidence that several lumenal proteins, including components of the photosynthetic chain, contain one or several disulfide bonds (Kieselbach, 2013; Table 1). However the functional importance of the disulfide(s) has only been examined for a small number of these proteins. The molecular mass of disulfide bonded proteins in the thylakoid lumen range from 10 to $55 \mathrm{kDa}$ and the disulfide bond forming cysteines do not appear to occur in a motif. This is at contrast with most of the disulfide bonded molecules in the mitochondrial IMS which are small proteins $(6-18 \mathrm{kDa})$ containing two disulfide bonds in either a $\mathrm{C}(X)_{3} \mathrm{C}$ or a $\mathrm{C}(X)_{9} \mathrm{C}$ motif (Sideris and Tokatlidis, 2010). Interestingly, while disulfide bond reduction in stroma-localized targets serves to activate several enzymes (Meyer et al., 2009), it appears that it is the reverse reaction, i.e., disulfide bond formation, that is required for the activities of several lumen-resident proteins (Buchanan and Luan, 2005).

In bacteria, the requirement for disulfide bond reduction in the periplasmic space was established mainly through studies of cytochrome $c$ maturation. Cytochromes $c$ are metalloproteins with one or several hemes covalently linked to a $\mathrm{CXXCH}$ motif on the protein (Thony-Meyer, 1997; Hamel et al., 2009). The need for a disulfide-reducing activity in the context of bacterial cytochrome $c$ assembly seemed obvious because the periplasmic space is also the compartment where cysteine-containing proteins are oxidized by dedicated catalysts (Denoncin and Collet, 2013). The current 
Table 1 | Lumenal disulfide bonded proteins in Arabidopsis.

\begin{tabular}{|c|c|c|c|}
\hline protein & Gene locus & Classification & Function \\
\hline PsbO1 (2 cys, 1 SS) & AT5G66570 & PSII subunit & Oxygen evolution \\
\hline PSI-N (4 cys, 2 SS) & AT5G64040 & PSI subunit & Regulation of PSI activity? \\
\hline Rieske (2 cys, 1 SS) & AT4G03280 & Cytochrome $b_{6} f$ subunit & Electron transfer in $b_{6} f$ complex \\
\hline FKBP13 (4 cys, 2 SS) & AT5G45680 & Peptidyl-prolyl cis-trans isomerase & Unknown \\
\hline FKBP20-2 (2 cys, 1 SS) & AT3G60370 & Peptidyl-prolyl cis-trans isomerase & PSII supercomplex assembly \\
\hline $\operatorname{PrxQ}(2$ cys, $1 \mathbf{S S})$ & AT3G26060 & Peroxiredoxin & Unknown \\
\hline $\operatorname{VDE}(13$ cys, $\geq \mathbf{4} \mathbf{~ S S})$ & AT1G08550 & Violaxanthin de-epoxidase & Photoprotection \\
\hline TL20.3 (4 cys, $\geq 1$ SS) & AT1G12250 & Pentapeptide repeat & Unknown \\
\hline TL29 (2 cys, 1 SS) & AT4G09010 & Ascorbate peroxidase & Unknown \\
\hline CtD1 (5 cys, $\geq 1 \mathrm{SS}$ ) & AT4G17740 & Protease & Processing of D1 subunit \\
\hline CtD1-like (4 cys, $\geq 1$ SS) & AT5G46390 & Protease & Unknown \\
\hline Deg5 (2 cys, 1 SS) & AT4G18370 & Protease & Degradation of lumenal proteins? \\
\hline
\end{tabular}

The number of lumenal cysteines (cys) that can form an intramolecular disulfide and the number of disulfides (SS) are indicated in parenthesis. The number of disulfide

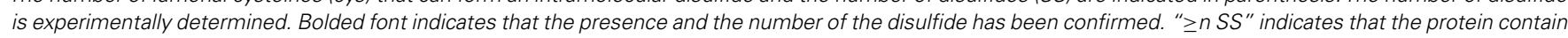

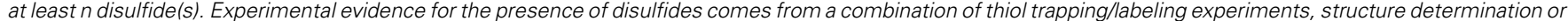

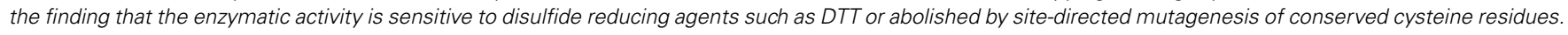

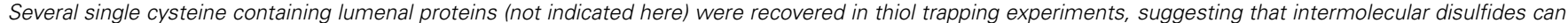
be formed. See references (Hall et al., 2010; Hall, 2012; Kieselbach, 2013).

view is that the $\mathrm{CXXCH}$ motif is oxidized after translocation of apocytochromes $c$ to the periplasmic space and then reduced by a reducing pathway to provide free sulfhydryls for ligation with heme (Bonnard et al., 2010; Sanders et al., 2010). This question had received little attention in the context of plastid cytochromes $c$ because thiol-disulfide chemistry as a catalyzed process was not believed to take place in the thylakoid lumen, the compartment where heme attachment to apocytochromes $c$ occurs. The discovery of trans-thylakoid redox pathways controlling disulfide bond formation and reduction in Chlamydomonas and Arabidopsis has now changed this perception.

\section{TWO DISULFIDE-REDUCING PATHWAYS OPERATE IN THE LUMEN}

A central component of the bacterial disulfide-reducing pathways is the thiol-disulfide oxido-reductase of the DsbD family (Cho and Collet, 2013). In bacteria, members of this family (CcdA, DsbD, $\mathrm{ScsB}$ ) are cytoplasmic membrane proteins conveying reducing power from the cytosol to the active sites of several target molecules in the periplasm. Reducing power is transferred across the cytoplasmic membrane through sequential thiol-disulfide exchanges (Cho and Collet, 2013). One target required for cytochrome $c$ assembly is the membrane-bound, periplasm-facing, thioredoxinlike protein $(\mathrm{CcmG} / \mathrm{ResA} / \mathrm{CcsX})$. CcmG/ResA/CcsX is postulated to reduce a disulfide in the $\mathrm{CXXCH}$ heme-binding site of apocytochrome $c$ prior to heme ligation to the cysteines (Bonnard etal., 2010; Sanders etal., 2010). In the plastid lumen, the involvement of a disulfide-reducing pathway was first suspected based on the presence of CCDA, an ortholog of bacterial CcdA from the DsbD family, at the thylakoid membrane (Page et al., 2004; Motohashi and Hisabori, 2010; Figure 1). Another component in the plastid is HCF164 (High Chlorophyll Fluorescence), a membrane-anchored, lumen-facing, thioredoxin-like protein with similarity to bacterial CcmG/ResA/CcsX (Lennartz et al., 2001; Figure 1). The disulfide-reducing activity of HCF164 was inferred from the fact that a recombinant form of its lumenal domain displays disulfide reductase activity in vitro (Lennartz et al., 2001; Motohashi and Hisabori, 2006). Loss of function of CCDA or HCF164 in Arabidopsis produces a photosyntheticdeficient phenotype due to a defect in cytochrome $b_{6} f$ complex assembly (Lennartz et al., 2001; Page et al., 2004). However, the biochemical activity and site of action of CCDA and HCF164 in the assembly process remained unknown until the characterization of the Chlamydomonas ccs4 and ccs5 mutants (Xie et al., 1998). The ccs4 and ccs5 mutants (ccs for cytochrome c synthesis), which are partially photosynthetic deficient, display a block in the conversion of apo to holoform of lumen resident cytochromes $c$, namely membrane-bound cytochrome 


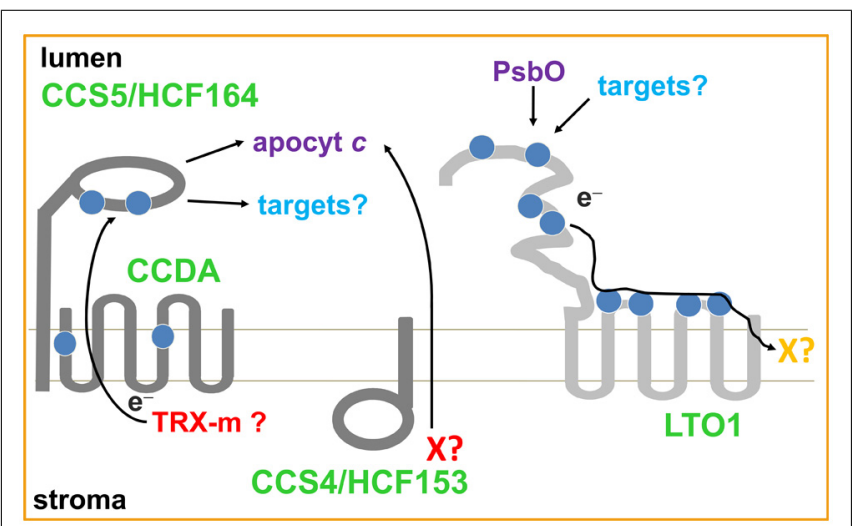

FIGURE 1 | Trans-thylakoid thiol metabolizing pathways. The disulfide-reducing (CCDA, CCS5/HCF164 and CCS4/HCF153) and thiol-oxidizing pathways (LTO1) are represented in dark and light grey, respectively. Cysteines are represented as blue balls. Electron $\left(e^{-}\right)$routes are indicated by arrows. The topology of CCS4/HCF164 is hypothetical but the positive-inside rule predicts a stromal localization for the C-terminal domain of the protein. CCS5/HCF164 is a membrane anchored lumen-facing thioredoxin-like protein. LTO1 contains a VKOR-like membrane domain and a lumen-facing thioredoxin-like domain. Stromal reductants for the disulfide reducing pathways are indicated in red. The electron acceptor for the thiol-oxidizing pathway is indicated in orange. Thioredoxin- $m$ (Trx-m) is the possible reductant for the CCDA-CCS5/HCF164 pathway. The CCS4/HCF153 pathway might be involved in the transport of a reductant such as glutathione or ascorbate (X?). The final electron acceptor of the LTO1-dependent pathway is not known and is postulated to be a phylloquinone (X?). CCDA and LTO1 topology was deduced using PhoA/LacZ topological reporters (Page et al., 2004; Feng et al., 2011; Karamoko etal., 2011). The thylakoid membrane is shown in light grey.

$f$ and soluble cytochrome $c_{6}$. The ccs mutants are deficient for cytochrome $b_{6} f$ assembly, which is dependent on cytochrome $f$, the thylakoid membrane-bound cytochrome $c$. This assembly defect is at the step of heme attachment to apocytochromes $c$, a chemical reaction taking place in the thylakoid lumen (Howe and Merchant, 1992, 1993, 1994; Xie et al., 1998). The Chlamydomonas CCS5 gene was cloned and shown to encode a thioredoxin-like protein with similarity to Arabidopsis HCF164 (Gabilly et al., 2010; Figure 1). Arabidopsis HCF164 can complement the cytochrome $c$ assembly defect when expressed from the plastid genome of the Chlamydomonas ccs5 mutant, demonstrating that HCF164 and CCS5 are functionally equivalent. This also suggests that the defect in cytochrome $b_{6} f$ assembly in the Arabidopsis hcf164 mutant is caused by a block in the conversion of apo to holocytochrome $f$. The role for CCS5 as an apocytochrome $c$ disulfide reductase in cytochrome $c$ assembly was formulated based on the following findings: (a) the ccs5-null mutant can be chemically rescued in vivo by application of exogenous thiols (such as DTT); (b) CCS5 interacts with plastid apocytochromes $c$ in yeast two-hybrid assays; and c) a recombinant form of CCS5 is able to reduce a disulfide bonded $\mathrm{CXXCH}$ in apocytochrome $c$ in vitro (Gabilly et al., 2010). The proposed model is that the thiol-disulfide oxido-reductase CCDA and the thioredoxin-like protein CCS5/HCF164 define a trans-thylakoid pathway for the delivery of reductants from the stroma to the lumen (Lennartz et al., 2001; Page etal., 2004; Motohashi and Hisabori, 2006;
Gabilly et al., 2010; Motohashi and Hisabori, 2010; Gabilly et al., 2011). This pathway is required for cytochrome $b_{6} f$ assembly but it is conceivable that it also acts as the transducer of reducing power to regulate the thiol-dependent activity of other lumenal targets (Motohashi and Hisabori, 2006). For instance, in vitro and in organello experiments showed that the reduction of the two disulfides in PSI-N (Table 1), a subunit of photosystem I (PSI), is dependent upon HCF164. The source of reducing power on the stromal side is not known but in organello experiments support the role of the thioredoxin $\operatorname{Trx}-m$ as a possible electron donor to CCDA and HCF164 (Motohashi and Hisabori, 2010).

The Chlamydomonas CCS4 gene encodes a small protein with limited similarity to Arabidopsis HCF153, a thylakoid membrane anchored protein required for the assembly of the cytochrome $b_{6} f$ complex (Lennartz et al., 2006; Gabilly et al., 2011; Figure 1). The CCS4 protein contains an N-terminal hydrophobic stretch that could serve as a membrane anchor and a C-terminal domain rich in charged residues. On the basis of the positive-inside rule that governs the topology of bacterial and thylakoid membrane proteins (von Heijne, 1989; Gavel et al., 1991), the C-terminal domain of CCS4 is predicted to be exposed to the stromal side of the thylakoid membrane. Surprisingly, CCS4 does not display any motif or residue (such as cysteines) suggesting a role in thiol-based redox chemistry. Yet, the thiol-dependent photosynthetic rescue of the $\operatorname{ccs} 4$ mutant and the suppression of the ccs4 phenotype by ectopic expression of CCDA, the thiol/disulfide oxido-reductase of the DsbD family, at the thylakoid membrane, confirms the activity of CCS4 in a disulfide-reducing pathway for cytochrome $c$ assembly. Moreover, the CCDA-dependent suppression of the ccs 4 mutant confirms the placement role of CCDA in plastid cytochrome $c$ maturation. Indeed, earlier studies in Arabidopsis supported, but did not establish, the requirement of plastid CCDA in the conversion of apo- to holocytochromes $c$ (Page et al., 1997; Motohashi and Hisabori, 2010). Interestingly, the ccs4-null ccs5-null double mutant displays a synthetic phenotype characterized by a complete loss of $b_{6}$ fassembly, an indication that CCS4 and CCS5 are redundant. This functional redundancy suggests that CCS4 might control a different disulfide-reducing pathway than the CCS5/HCF164 dependent one (Figure 1). The CCDA-dependent suppression of the $c c s 4$ mutant can be explained by a compensatory effect due to enhanced expression of the thiol-disulfide oxido-reductase CCDA. At the present time, the activity of CCS4 in such a pathway remains elusive. While the CCDA dependent pathway relies on the transfer of reductants through sequential thiol-disulfide exchanges, it is conceivable that CCS4 controls the transport across the thylakoid membrane of a molecule acting as a reducing agent (Figure 1). In bacteria, operation of such redundant routes for export of reductant to the periplasm have been postulated (Pittman et al., 2005). The nature of the exported reductant can only be speculated upon but glutathione or ascorbate is an obvious candidate. While the presence of glutathione in the thylakoid lumen remains to be established, ascorbate is known to function in this compartment as an alternative electron donor to PSII and a co-factor for violaxanthin de-epoxidase (VDE) dependent photoprotection (Tóth et al., 2013). 


\section{DISCOVERY OF A DISULFIDE-FORMING ENZYME IN THE THYLAKOID LUMEN}

In the periplasmic space of most proteobacteria, the thioloxidizing pathway consists of a disulfide bond catalyzing system defined by soluble DsbA and membrane-bound DsbB (Denoncin and Collet, 2013). DsbA catalyzes disulfide bridge formation in cysteine-containing substrates that are translocated across the membrane into the periplasmic space. DsbB operates by recycling reduced DsbA to its oxidized form with transfer of electrons to quinones, which are membrane-soluble redox carriers in the respiratory chain. The fact that no DsbA or DsbB-like enzymes can be detected in the genomes of photosynthetic eukaryotes or Cyanobacteria, the presumed ancestors of the chloroplast, reinforced the view that disulfide bond formation did not take place in the thylakoid lumen. However, the operation of catalyzed disulfide bond formation in the lumen is supported by the finding that bacterial alkaline phosphatase (PhoA), an enzyme requiring two disulfide bonds for activity, and basic pancreatic trypsin inhibitor BPTI (aprotinin), a molecule containing three disulfide bonds, are active when targeted to this compartment in tobacco (Sone et al., 1997; Bally et al., 2008; Tissot et al., 2008). A novel class of disulfide-forming enzymes with similarity to VKOR (Vitamin K epoxide Oxidoreductase) was recently recognized in some bacterial phyla lacking the typical DsbAB components (including Cyanobacteria) and in all photosynthetic eukaryotes (Dutton et al., 2008; Singh etal., 2008; Grossman et al., 2010). VKOR is well studied for its involvement in the reduction of vitamin $\mathrm{K}$, a phylloquinone required as a co-factor for the $\gamma$-carboxylation of clotting factors in blood (Tie and Stafford, 2008). Recent work shows that the enzymatic activity of VKOR is also linked to oxidative folding of proteins in the ER lumen (Rishavy etal., 2011; Schulman et al., 2011). A first indication that the thylakoid lumen houses a thiol-oxidizing pathway came from the identification of a VKOR-like protein in Cyanobacteria (Singh et al., 2008). In vitro reconstitution of disulfide bond formation with the purified cyanobacterial enzyme demonstrated the sulfhydryl oxidase activity of the protein (Li et al., 2010). However, the localization of the protein at the thylakoid membrane and its relevant targets of action in the lumen were not documented (Singh etal., 2008). The identity of the thiol-oxidizing catalyst in the thylakoid lumen of plastids has now emerged through the discovery of LTO1 (Lumen Thiol Oxidoreductase 1), a thylakoid membrane protein containing a VKOR domain fused to a thioredoxin-like moiety (Figure 1; Furt etal., 2010; Feng etal., 2011; Karamoko etal., 2011). Topological studies using bacterial topological reporters established a lumenal location for the LTO1 domains carrying the redox motifs and conserved cysteines (Figure 1). Previous studies with bacterial VKOR-like proteins have demonstrated that the thioredoxin-like domain carries a DsbA-like activity while the VKOR-like central domain is functionally equivalent to DsbB (Singh etal., 2008; Dutton etal., 2010; Wang etal., 2011). In Arabidopsis, loss of LTO1 function is associated with a severe phototrophic growth defect (Karamoko et al., 2011; Lu et al., 2013). Measurements of the photosynthetic activity indicate that lto1 mutants display a limitation in the electron flow from Photosystem II (PSII). In accord with these measurements, lto1 mutants show a severe depletion of several of the structural subunits of
PSII (including subunits of the OEC) but no change in the accumulation of the cytochrome $b_{6} f$ complex or PSI and no defect in the activity of ATP synthase. In a yeast two-hybrid assay, the lumen-facing thioredoxin-like domain of LTO1 was shown to interact with PsbO, a lumenal PSII subunit in the OEC known to be disulfide bonded (Table 1). In vitro, the thioredoxin-like domain of LTO1 is able to introduce a disulfide bond in the PsbO target when recombinant forms of the molecules are used. Because the redox state of the sulfhydryls in PsbO was shown to be a determinant for the stability of this subunit and also for PSII accumulation (Burnap et al., 1994; Nikitina et al., 2008; Hall et al., 2010), it is likely that loss of disulfide bond formation in PsbO in the lto 1 mutants accounts for the PSII assembly defect. It is not known if the ability of LTO1 to form a disulfide bond in PsbO is linked to the import of this protein into the lumen, similarly to the Mia40-dependent pathway in mitochondria (Herrmann and Riemer, 2012). In organello import experiments showed that PsbO translocates at a different site than PsbP, another subunit of the PSII OEC (Hashimoto et al., 1997). It is plausible that this translocation step is assisted by LTO1 but this awaits experimental testing.

The final electron acceptor of the LTO1-dependent disulfide bond forming pathway is currently unknown (Figure 1). It is likely to be a phylloquinone based on the fact that the Arabidopsis protein reduces phylloquinone in an in vitro enzymatic assay (Furt et al., 2010). The role of phylloquinone as a structural cofactor tightly bound to PSI is well documented (Brettel, 1997). However, the occurrence of a pool of phylloquinone that is not bound to PSI suggests phylloquinone might participate in redox processes in addition to the known electron transfer reactions through PSI (Gross et al., 2006; Lohmann et al., 2006). This pool may act as an electron acceptor for the LTO1-dependent disulfide bond forming pathway in vivo.

\section{OUTLOOK}

It is conceivable that catalyzed thiol-oxidation in the lumen extends to other disulfide-bond containing targets in addition to PsbO (Table 1). In vitro experiments suggest that the thioredoxinlike domain of LTO1 is also able to catalyze the formation of the two disulfide bonds in FKBP13 (Lu et al., 2013), a peptidyl-prolyl cis-trans isomerase whose activity is dependent upon sulfhydryl oxidation (Gopalan etal., 2004; Table 1). It is not known if additional enzymes with sulfhydryl oxidase activity besides LTO1 also operate in the thylakoid lumen. In bacteria, the disulfidereducing pathway is also required to maintain the reduction state of periplasmic oxido-reductases that shuffle disulfide bonds that are incorrectly formed (Kadokura and Beckwith, 2010; Depuydt et al., 2011). The need for disulfide bond isomerization is critical for proteins containing more than two cysteines such as VDE, an enzyme involved in photoprotection whose activity depends on sulfhydryl oxidation (Kanervo et al., 2005; Table 1). Interestingly, the activity of recombinant VDE was initially reported to be low, presumably because of improper protein folding due to incorrect disulfide linkages (Bugos and Yamamoto, 1996; Hieber et al., 2002). Expression in the cytosol of a bacterial strain engineered for disulfide bond formation and isomerization resulted in a high level of VDE activity for the purified enzyme, an indication 
that isomerization of disulfide bond in the lumen is also likely to be required for yielding an active enzyme (Saga et al., 2010). It is possible that LTO1 exhibits this activity based on the finding that its thioredoxin-like domain is active as a disulfide-bond isomerase in vitro (Lu et al., 2013). Another thylakoid membrane protein displaying disulfide-bond isomerase activity in vitro is LQY1, an enzyme required for the repair and re-assembly of photodamaged PSII (Lu, 2011). However, it is not known if the active site of LQY1 faces the lumenal or stromal side of the thylakoid membrane. Further experimental work is required to establish the molecular identity of the missing redox components defining the thiol-metabolizing pathways, identify their relevant targets of action and understand how they control photosynthesis or other processes in the thylakoid lumen.

\section{ACKNOWLEDGMENTS}

This work is supported by a National Science Foundation grant (MCB-0920062) to Patrice P. Hamel. We thank Dr. Lamb and Dr. Ruiz for critical reading of the manuscript.

\section{REFERENCES}

Bally, J., Paget, E., Droux, M., Job, C., Job, D., and Dubald, M. (2008). Both the stroma and thylakoid lumen of tobacco chloroplasts are competent for the formation of disulphide bonds in recombinant proteins. Plant Biotechnol. J. 6, 46-61. doi: 10.1111/j.1467-7652.2007.00298.x

Bonnard, G., Corvest, V., Meyer, E. H., and Hamel, P. P. (2010). Redox processes controlling the biogenesis of c-type cytochromes. Antioxid. Redox Signal. 13, 1385-1401. doi: 10.1089/ars.2010.3161

Brettel, K. (1997). Electron transfer and arrangement of the redox cofactors in photosystem I. Biochim. Biophys. Acta 1318, 322-373. doi: 10.1016/S00052728(96)00112-0

Buchanan, B. B., and Luan, S. (2005). Redox regulation in the chloroplast thylakoid lumen: a new frontier in photosynthesis research. J. Exp. Bot. 56, 1439-1447. doi: 10.1093/jxb/eri158

Bugos, R. C., and Yamamoto, H. Y. (1996). Molecular cloning of violaxanthin deepoxidase from romaine lettuce and expression in Escherichia coli. Proc. Natl. Acad. Sci. USA 93, 6320-6325. doi: 10.1073/pnas.93.13.6320

Burnap, R. L., Qian, M., Shen, J. R., Inoue, Y., and Sherman, L. A. (1994). Role of disulfide linkage and putative intermolecular binding residues in the stability and binding of the extrinsic manganese-stabilizing protein to the photosystem II reaction center. Biochemistry 33, 13712-13718. doi: 10.1021/bi00250a023

Cho, S. H., and Collet, J. F. (2013). Many roles of the bacterial envelope reducing pathways. Antioxid. Redox Signal. 18, 1690-1698. doi: 10.1089/ars.2012.4962

Denoncin, K., and Collet, J. F. (2013). Disulfide bond formation in the bacterial periplasm: major achievements and challenges ahead. Antioxid. Redox Signal. 19, 63-71. doi: 10.1089/ars.2012.4864

Depuydt, M., Messens, J., and Collet, J.-F. (2011). How proteins form disulfide bonds. Antioxid. Redox Signal. 15, 49-66. doi: 10.1089/ars.2010.3575

Dutton, R. J., Boyd, D., Berkmen, M., and Beckwith, J. (2008). Bacterial species exhibit diversity in their mechanisms and capacity for protein disulfide bond formation. Proc. Natl. Acad. Sci. USA 105, 11933-11938. doi: 10.1073/pnas.0804621105

Dutton, R. J., Wayman, A., Wei, J.-R., Rubin, E. J., Beckwith, J., and Boyd, D. (2010). Inhibition of bacterial disulfide bond formation by the anticoagulant warfarin. Proc. Natl. Acad. Sci. USA 107, 297-301. doi: 10.1073/pnas.0912952107

Feng, W. K., Wang, L., Lu, Y., and Wang, X. Y. (2011). A protein oxidase catalysing disulfide bond formation is localized to the chloroplast thylakoids. FEBS J. 278, 3419-3430. doi: 10.1111/j.1742-4658.2011.08265.x

Furt, F., Oostende, C., Widhalm, J. R., Dale, M. A., Wertz, J., and Basset, G. J. (2010). A bimodular oxidoreductase mediates the specific reduction of phylloquinone (vitamin K) in chloroplasts. Plant J. 64, 38-46. doi: 10.1111/j.1365-313X.2010.04305.x

Gabilly, S. T., Dreyfuss, B. W., Karamoko, M., Corvest, V., Kropat, J., Page, M. D., et al. (2010). CCS5, a thioredoxin-like protein involved in the assembly of plastid c-type cytochromes. J. Biol. Chem. 285, 29738-29749. doi: 10.1074/jbc.M109.099069
Gabilly, S. T., Kropat, J., Karamoko, M., Page, M. D., Nakamoto, S. S., Merchant, S. S., et al. (2011). A novel component of the disulfide-reducing pathway required for cytochrome c assembly in plastids. Genetics 187, 793-802. doi: 10.1534/genetics.110.125369

Gavel, Y., Steppuhn, J., Herrmann, R., and Von Heijne, G. (1991). The 'positiveinside rule' applies to thylakoid membrane proteins. FEBS Lett. 282, 41-46. doi: 10.1016/0014-5793(91)80440-E

Gopalan, G., He, Z., Balmer, Y., Romano, P., Gupta, R., Héroux, A., et al. (2004). Structural analysis uncovers a role for redox in regulating FKBP13, an immunophilin of the chloroplast thylakoid lumen. Proc. Natl. Acad. Sci. USA 101, 13945-13950. doi: 10.1073/pnas.0405240101

Gross, J., Cho, W. K., Lezhneva, L., Falk, J., Krupinska, K., Shinozaki, K., et al. (2006). A plant locus essential for phylloquinone (vitamin K1) biosynthesis originated from a fusion of four eubacterial genes. J. Biol. Chem. 281, 17189-17196. doi: 10.1074/jbc.M601754200

Grossman, A. R., Karpowicz, S. J., Heinnickel, M., Dewez, D., Hamel, B., Dent, R., et al. (2010). Phylogenomic analysis of the Chlamydomonas genome unmasks proteins potentially involved in photosynthetic function and regulation. Photosynth. Res. 106, 3-17. doi: 10.1007/s11120-010-9555-7

Hall, M. (2012). The Chloroplast Lumen - New Insights into Thiol Redox Regulation and Functions of Lumenal Proteins. Ph.D. thesis, Umeå University, Sweden.

Hall, M., Mata-Cabana, A., Akerlund, H. E., Florencio, F. J., Schroder, W. P., Lindahl, M., et al. (2010). Thioredoxin targets of the plant chloroplast lumen and their implications for plastid function. Proteomics 10, 987-1001. doi: 10.1002/pmic.200900654.

Hamel, P., Corvest, V., Giege, P., and Bonnard, G. (2009). Biochemical requirements for the maturation of mitochondrial c-type cytochromes. Biochim. Biophys. Acta 1793, 125-138. doi: 10.1016/j.bbamcr.2008.06.017

Hashimoto, A., Ettinger, W. F., Yamamoto, Y., and Theg, S. M. (1997). Assembly of newly imported oxygen-evolving complex subunits in isolated chloroplasts: sites of assembly and mechanism of binding. Plant Cell 9, 441-452. doi:10.1105/tpc.9.3.441

Herrmann, J. M., and Riemer, J. (2012). Mitochondrial disulfide relay: redoxregulated protein import into the intermembrane space. J. Biol. Chem. 287, 4426-4433. doi: 10.1074/jbc.R111.270678

Hieber, D., Bugos, R., Verhoeven, A., and Yamamoto, H. (2002). Overexpression of violaxanthin de-epoxidase: properties of C-terminal deletions on activity and $\mathrm{pH}$ dependent lipid binding. Planta 214, 476-483. doi: 10.1007/s00425-001-0704-2

Howe, G., and Merchant, S. (1992). The biosynthesis of membrane and soluble plastidic c-type cytochromes of Chlamydomonas reinhardtii is dependent on multiple common gene products. EMBO J. 11, 2789-2801.

Howe, G., and Merchant, S. (1993). Maturation of thylakoid lumen proteins proceeds post-translationally through an intermediate in vivo. Proc. Natl. Acad. Sci. USA 90, 1862-1866. doi: 10.1073/pnas.90.5.1862

Howe, G., and Merchant, S. (1994). Role of heme in the biosynthesis of cytochrome c6. J. Biol. Chem. 269, 5824-5832.

Kadokura, H., and Beckwith, J. (2010). Mechanisms of oxidative protein folding in the bacterial cell envelope. Antioxid. Redox Signal. 13, 1231-1246. doi: 10.1089/ars.2010.3187

Kanervo, E., Suorsa, M., and Aro, E.-M. (2005). Functional flexibility and acclimation of the thylakoid membrane. Photochem Photobiol. Sci. 4, 1072-1080. doi: $10.1039 / \mathrm{b} 507866 \mathrm{k}$

Karamoko, M., Cline, S., Redding, K., Ruiz, N., and Hamel, P. P. (2011). Lumen thiol oxidoreductase1, a disulfide bond-forming catalyst, is required for the assembly of photosystem II in Arabidopsis. Plant Cell 23, 4462-4475. doi: 10.1105/tpc. 111.089680

Kieselbach, T. (2013). Oxidative folding in chloroplasts. Antioxid. Redox Signal. 19, 72-82. doi: 10.1089/ars.2012.4582

Kieselbach, T., and Schröder, W. (2003). The proteome of the chloroplast lumen of higher plants. Photosyn. Res. 78, 249-264. doi: 10.1023/B:PRES.0000006913.86689.f1

Lennartz, K., Bossmann, S., Westhoff, P., Bechtold, N., and Meierhoff, K. (2006). HCF153, a novel nuclear-encoded factor necessary during a post-translational step in biogenesis of the cytochrome bf complex. Plant J. 45, 101-112. doi: 10.1111/j.1365-313X.2005.02605.x

Lennartz, K., Plücken, H., Seidler, A., Westhoff, P., Bechtold, N., and Meierhoff, K. (2001). HCF164 encodes a thioredoxin-like protein involved in the biogenesis of the cytochrome b6f complex in Arabidopsis. Plant Cell 13, 2539-2351. 
Li, W., Schulman, S., Dutton, R. J., Boyd, D., Beckwith, J., and Rapoport, T. A. (2010). Structure of a bacterial homologue of vitamin K epoxide reductase. Nature 463, 507-512. doi: 10.1038/nature08720

Lohmann, A., Schottler, M. A., Brehelin, C., Kessler, F., Bock, R., Cahoon, E. B., et al. (2006). Deficiency in phylloquinone (vitamin K1) methylation affects prenyl quinone distribution, photosystem I abundance, and anthocyanin accumulation in the Arabidopsis AtmenG mutant. J. Biol. Chem. 281, 40461-40472. doi: 10.1074/jbc.M609412200

Lu, Y. (2011). The occurrence of a thylakoid-localized small zinc finger protein in land plants. Plant Signal. Behav. 6, 1881-1885. doi: 10.4161/psb.6.12. 18022

Lu, Y., Wang, H. R., Li, H., Cui, H. R., Feng, Y. G., and Wang, X. Y. (2013) A chloroplast membrane protein LTO1/AtVKOR involving in redox regulation and ROS homeostasis. Plant Cell Rep. 32, 1427-1440. doi: 10.1007/s00299-0131455-9

Meyer, Y., Buchanan, B. B., Vignols, F., and Reichheld, J.-P. (2009). Thioredoxins and glutaredoxins: unifying elements in redox biology. Annu. Rev. Genet. 43, 335-367. doi: 10.1146/annurev-genet-102108-134201

Motohashi, K., and Hisabori, T. (2006). HCF164 receives reducing equivalents from stromal thioredoxin across the thylakoid membrane and mediates reduction of target proteins in the thylakoid lumen. J. Biol. Chem. 281, 35039-35047. doi: 10.1074/jbc.M605938200

Motohashi, K., and Hisabori, T. (2010). CcdA is a thylakoid membrane protein required for the transfer of reducing equivalents from stroma to thylakoid lumen in the higher plant chloroplast. Antioxid. Redox Signal. 13, 1169-1176. doi: 10.1089/ars.2010.3138

Nikitina, J., Shutova, T., Melnik, B., Chernyshov, S., Marchenkov, V., Semisotnov, G., et al. (2008). Importance of a single disulfide bond for the PsbO protein of photosystem II: protein structure stability and soluble overexpression in Escherichia coli. Photosynth. Res. 98, 391-403. doi: 10.1007/s11120-008-9327-9

Page, M. D., Saunders, N. F., and Ferguson, S. J. (1997). Disruption of the Pseudomonas aeruginosa dipZ gene, encoding a putative protein-disulfide reductase, leads to partial pleiotropic deficiency in c-type cytochrome biogenesis. Microbiology 143, 3111-3122. doi: 10.1099/00221287-143-10-3111

Page, M. L. D., Hamel, P. P., Gabilly, S. T., Zegzouti, H., Perea, J. V., Alonso, J. M., et al. (2004). A homolog of prokaryotic thiol disulfide transporter CcdA is required for the assembly of the cytochrome b6f complex in Arabidopsis chloroplasts. J. Biol. Chem. 279, 32474-32482. doi: 10.1074/jbc.M404285200

Pittman, M. S., Robinson, H. C., and Poole, R. K. (2005). A bacterial glutathione transporter (Escherichia coli CydDC) exports reductant to the periplasm. J. Biol. Chem. 280, 32254-32261. doi: 10.1074/jbc.M503075200

Rishavy, M. A., Usubalieva, A., Hallgren, K. W., and Berkner, K. L. (2011). Novel insight into the mechanism of the vitamin $\mathrm{K}$ oxidoreductase (VKOR). J. Biol. Chem. 286, 7267-7278. doi: 10.1074/jbc.M110.172213

Roose, J. L., Frankel, L. K., and Bricker, T. M. (2011). Developmental defects in mutants of the PsbP domain protein 5 in Arabidopsis thaliana. PLoS ONE 6:e28624. doi: 10.1371/journal.pone.0028624

Saga, G., Giorgetti, A., Fufezan, C., Giacometti, G. M., Bassi, R., and Morosinotto, T. (2010). Mutation analysis of violaxanthin de-epoxidase identifies substratebinding sites and residues involved in catalysis. J. Biol. Chem. 285, 23763-23770. doi: 10.1074/jbc.M110.115097

Sanders, C., Turkarslan, S., Lee, D.-W., and Daldal, F. (2010). Cytochrome c biogenesis: the Ccm system. TIM 18, 266-274. doi: 10.1016/j.tim.2010.03.006
Schulman, S., Wang, B., Li, W., and Rapoport, T. A. (2011). Vitamin K epoxide reductase prefers ER membrane-anchored thioredoxin-like redox partners. Proc. Natl. Acad. Sci. USA 107, 15027-15032. doi: 10.1073/pnas.1009972107

Sideris, D. P., and Tokatlidis, K. (2010). Oxidative protein folding in the mitochondrial intermembrane space. Antioxid. Redox Signal. 13, 1189-1204. doi: 10.1089/ars.2010.3157

Singh, A. K., Bhattacharyya-Pakrasi, M., and Pakrasi, H. B. (2008). Identification of an atypical membrane protein involved in the formation of protein disulfide bonds in oxygenic photosynthetic organisms. J. Biol. Chem. 283, 15762-15770. doi: 10.1074/jbc.M800982200

Sone, M., Kishigami, S., Yoshihisa, T., and Ito, K. (1997). Roles of disulfide bonds in bacterial alkaline phosphatase. J. Biol. Chem. 272, 6174-6178. doi: 10.1074/jbc.272.10.6174

Thony-Meyer, L. (1997). Biogenesis of respiratory cytochromes in bacteria. Microbiol. Mol. Biol. Rev. 61, 337-376.

Tie, J. K., and Stafford, D. W. (2008). Structure and function of vitamin K epoxide reductase. Vitam. Horm. 78, 103-130. doi: 10.1016/S0083-6729(07)00006-4

Tissot, G., Canard, H., Nadai, M., Martone, A., Botterman, J., and Dubald, M. (2008). Translocation of aprotinin, a therapeutic protease inhibitor, into the thylakoid lumen of genetically engineered tobacco chloroplasts. Plant Biotechnol. J. 6, 309-320. doi: 10.1111/j.1467-7652.2008.00321.x

Tóth, S. Z., Schansker, G., and Garab, G. (2013). The physiological roles and metabolism of ascorbate in chloroplasts. Physiol. Plant. 148, 161-175. doi: $10.1111 /$ ppl.12006

von Heijne, G. (1989). Control of topology and mode of assembly of a polytopic membrane protein by positively charged residues. Nature 341, 456-458. doi: $10.1038 / 341456 \mathrm{a} 0$

Wang, X., Dutton, R. J., Beckwith, J., and Boyd, D. (2011). Membrane topology and mutational analysis of Mycobacterium tuberculosis VKOR, a protein involved in disulfide bond formation and a homologue of human vitamin $\mathrm{K}$ epoxide reductase. Antioxid. Redox Signal. 14, 1413-1420. doi: 10.1089/ars.2010.3558

Xie, Z., Culler, D., Dreyfuss, B. W., Kuras, R., Wollman, F. A., Girard-Bascou, J., et al. (1998). Genetic analysis of chloroplast c-type cytochrome assembly in Chlamydomonas reinhardtii: one chloroplast locus and at least four nuclear loci are required for heme attachment. Genetics 148, 681-692.

Conflict of Interest Statement: The authors declare that the research was conducted in the absence of any commercial or financial relationships that could be construed as a potential conflict of interest.

Received: 15 October 2013; paper pending published: 29 October 2013; accepted: 04 November 2013; published online: 27 November 2013.

Citation: Karamoko M, Gabilly ST and Hamel PP (2013) Operation of transthylakoid thiol-metabolizing pathways in photosynthesis. Front. Plant Sci. 4:476. doi: 10.3389/fpls.2013.00476

This article was submitted to Plant Physiology, a section of the journal Frontiers in Plant Science.

Copyright (C) 2013 Karamoko, Gabilly and Hamel. This is an open-access article distributed under the terms of the Creative Commons Attribution License (CC BY). The use, distribution or reproduction in other forums is permitted, provided the original author(s) or licensor are credited and that the original publication in this journal is cited, in accordance with accepted academic practice. No use, distribution or reproduction is permitted which does not comply with these terms. 